\title{
A neurodegenerative vascular burden index and the impact on cognition
}

\author{
Sebastian Heinzel ${ }^{1}{ }^{*}$, Inga Liepelt-Scarfone ${ }^{1,2}$, Benjamin Roeben ${ }^{1}$, Isabella Nasi-Kordhishti ${ }^{1}$, \\ Ulrike Suenkel ${ }^{1}$, Isabel Wurster ${ }^{1}$, Kathrin Brockmann ${ }^{1}$, Andreas Fritsche ${ }^{3}$, Raphael Niebler ${ }^{4,5}$, \\ Florian G. Metzger ${ }^{4,5}$, Gerhard W. Eschweiler ${ }^{4,5}$, Andreas J. Fallgatter ${ }^{4}$, Walter Maetzler ${ }^{1}$ and Daniela Berg ${ }^{1,2}$ \\ 1 Department of Neurodegeneration, Hertie Institute for Clinical Brain Research (HIH), University of Tübingen, Tübingen, Germany \\ ${ }^{2}$ German Center for Neurodegenerative Diseases (DZNE), Tübingen, Germany \\ ${ }^{3}$ Department of Internal Medicine IV, University of Tübingen, Tübingen, Germany \\ ${ }^{4}$ Department of Psychiatry and Psychotherapy, University of Tübingen, Tübingen, Germany \\ ${ }^{5}$ Geriatric Center, University Hospital Tübingen, Tübingen, Germany
}

\section{Edited by:}

Isidro Ferrer, University of Barcelona,

Spain

Reviewed by:

Isidro Ferrer, University of Barcelona

Spain

Thomas Polak, University Clinic

Würzburg, Germany

\section{*Correspondence:}

Sebastian Heinzel, Department of

Neurodegeneration, Hertie Institute

for Clinical Brain Research (HIH),

University of Tübingen,

Hoppe-Seyler-Strasse 3, 72076

Tübingen, Germany

e-mail: sebastian.heinzel@med.

uni-tuebingen.de
A wide range of vascular burden factors has been identified to impact vascular function and structure as indicated by carotid intima-media thickness (IMT). On the basis of their impact on IMT, vascular factors may be selected and clustered in a vascular burden index (VBI). Since many vascular factors increase the risk of Alzheimer's disease (AD), a multifactorial neurodegenerative $\mathrm{VBI}$ may be related to early pathological processes in $\mathrm{AD}$ and cognitive decline in its preclinical stages. We investigated an elderly cohort at risk for neurodegeneration (TREND study, $n=1102$ ) for the multifactorial influence of vascular burden factors on IMT measured by ultrasound. To create a VBI for this cohort, vascular factors and their definitions (considering medical history, medication, and/or blood marker data) were selected based on their statistical effects on IMT in multiple regressions including age and sex. The impact of the VBI on cognitive performance was assessed using the Trail-Making Test (TMT) and the consortium to establish a registry for Alzheimer's disease (CERAD) neuropsychological battery. IMT was significantly predicted by age (standardized $\beta=0.26)$, sex $(0.09$; males $>$ females) and the factors included in the VBI: obesity (0.18), hypertension (0.14), smoking (0.08), diabetes (0.07), and atherosclerosis (0.05), whereas other cardiovascular diseases or hypercholesterolemia were not significant. Individuals with 2 or more VBI factors compared to individuals without had an odds ratio of 3.17 regarding overly increased IMT $(\geq 1.0 \mathrm{~mm})$. The VBI showed an impact on executive control [log (TMT B-A), $p=0.047]$ and a trend toward decreased global cognitive function (CERAD total score, $p=0.057$ ) independent of age, sex, and education. A VBI established on the basis of IMT may help to identify individuals with overly increased vascular burden linked to decreased cognitive function indicating neurodegenerative processes. The longitudinal study of this risk cohort will reveal the value of the VBI as prodromal marker for cognitive decline and $A D$.

Keywords: vascular burden, carotid intima-media thickness, trail-making test, CERAD neuropsychological battery, prodromal marker, neurodegeneration, Alzheimer's disease, Tübinger evaluation of risk factors for early detection of neurodegeneration (TREND) study

\section{INTRODUCTION}

The circulatory and metabolic functions of the vascular system underlying the reliable supply of oxygen and nutrients can be compromised by a wide range of factors. Pathological processes as well as lifestyle factors may affect vascular structure and function during life span. For instance, cardiovascular diseases, atherosclerosis, stroke, diabetes, hypertension, as well as smoking, obesity, hypercholesterolemia, and lack of physical activity have been associated with altered vascular structure (Salonen and Salonen, 1991; Folsom et al., 1994; Bots et al., 1997; van Popele et al., 2001; Urbina et al., 2002; Kotsis et al., 2006; Baldassarre et al., 2010; Engelen et al., 2012; Niu et al., 2013; van den Oord et al., 2013). Specifically, these studies showed an association of these vascular factors with structural increases in the intima-media thickness (IMT) of the common carotid artery (CCA). Increased IMT has been shown to serve as an indicator of vascular age (Stein et al., 2004) and arterial stiffness (van Popele et al., 2001), and to represent a strong predictor of clinical cardiovascular events (Lorenz et al., 2007). Clustering of multiple factors into a cumulative vascular burden index (VBI) has been shown to predict IMT better than single factors do (Urbina et al., 2002; Baldassarre et al., 2010; Niu et al., 2013) suggesting a converging impact of multiple different factors on IMT.

Other indices have been established comprising (cardio)vascular as well as other factors, such as age and sex, to estimate the annual risk of stroke for patients with atrial fibrillation $\left(\mathrm{CHA}_{2} \mathrm{DS}_{2}\right.$-VASc score; Olesen et al., 2011) or the 10-year cardiovascular risk of an individual (Framingham risk score; D'Agostino et al., 2008). While these (cardio)vascular risk indices have a high validity and specificity for particular diseases, they may not 
indicate vascular burden as relevant for other pathologies, e.g., neurodegenerative disease such as Alzheimer's disease (AD).

For the risk evaluation of $\mathrm{AD}$ an individual quantification of vascular burden mediated through multiple vascular factors associated with $\mathrm{AD}$ is crucial for two main reasons. (1) Chronic brain hypoperfusion leading to cerebral hypoxia represents an important aspect of neurodegenerative processes in $\mathrm{AD}$ and vascular dementia (Breteler, 2000; Zlokovic, 2005; de la Torre, 2012b), and often precedes the first cognitive symptoms by many years (Chao et al., 2010; de la Torre, 2012a). (2) Many vascular factors such as hypertension, diabetes, obesity, hypercholesterolemia, smoking, or stroke have been shown to increase the AD risk (Breteler, 2000; Honig et al., 2003; Kivipelto et al., 2005; Kivipelto and Solomon, 2006; Purnell et al., 2009; Cataldo et al., 2010) and the conversion from mild cognitive impairment (MCI) to AD (Li et al., 2011). A VBI may therefore be of particular importance for longitudinal studies, where vascular burden may predict $\mathrm{AD}$ or help to detect its early preclinical stages.

Decreased cognitive function as assessed using well-established neuropsychological tests is characteristic for MCI and AD and key to their diagnosis (Hort et al., 2010). Neurodegenerative processes as well as cognitive decline precede the clinical diagnosis of MCI and AD (Smith et al., 2012; Langbaum et al., 2013; Moghekar et al., 2013; Lim et al., 2014). Given the important role of vascular burden factors in the etiology of $\mathrm{AD}$, the impact of a VBI on cognitive function in non-demented elderly individuals may validate its relevance for the early detection of MCI and AD.

In the present study, we used vascular risk factors of $\mathrm{AD}$ showing an impact on IMT to establish a VBI in a large cohort of non-demented, elderly individuals [Tübinger evaluation of risk factors for early detection of neurodegeneration (TREND) study, $n=1102$, 51-84 years, (Hobert et al., 2011; Berg, 2012; Maetzler et al., 2012; Heinzel et al., 2013; Gaenslen et al., 2014)]. We hypothesized that this multifactorial VBI may contribute to neurodegenerative aging processes. To validate this link, we investigated this cohort at risk for neurodegeneration for the impact of the VBI on cognitive performance in neuropsychological tests.

\section{MATERIALS AND METHODS PARTICIPANTS}

All individuals included in the present analyses $(n=1102 ; 528$ female, 574 male) participated in the first follow up of the TREND study (March 2011 to April 2012), which represents the baseline assessment with regard to the IMT ultrasound measurements. The prospective and longitudinal TREND study aims to identify risk markers for the prediction of $\mathrm{AD}$ and Parkinson's disease (PD), respectively, in non-demented elderly individuals who are investigated every 2 years. Participants were partly selected due to the presence of prodromal risk markers of $\mathrm{AD}$ and $\mathrm{PD}$, respectively, including REM-sleep disorder, hyposmia, and/or depression. For a detailed outline of the TREND study, further inclusion and exclusion criteria and TREND baseline assessments, see Berg (2012). The study was approved by the ethical committee of the Medical Faculty of the University of Tübingen (Nr. 90/2009BO2). All procedures were in accordance with the Declaration of Helsinki in its latest version, and all participants gave written informed consent.

\section{VASCULAR BURDEN INDEX}

In order to establish a VBI for the TREND cohort, we first investigated $\mathrm{AD}$-associated vascular risk factors with different possible definitions (see below) for their effect size regarding IMT prediction. We then selected single factors and definitions yielding the best prediction of IMT (independent of age and sex) in multiple regression analyses (see Statistics). The cumulative number of these selected factors/definitions was used as VBI.

\section{VASCULAR BURDEN FACTORS}

Vascular risk factors, diseases and medication data of the TREND study were assessed with questionnaires and personal medical history interviews. The following factor definitions were included in the analyses (see also Table $\mathbf{1}$ ).

\section{Diabetes}

Life-time diagnosis of diabetes (factor label: medical history), additional intake of antidiabetic medication (medical history or medication), and glycated hemoglobin $\left(\mathrm{HbA}_{1 \mathrm{c}}\right)$ levels of $\mathrm{HbA}_{1 \mathrm{c}} \geq 6.5 \%$ (Kilpatrick et al., 2009) (medical history or medication or $\mathrm{HbA}_{1 \mathrm{c}}$ ) were considered as factors definitions.

\section{Hypertension}

Life-time diagnosis of hypertension (medical history), intake of anti-hypertensive medication alone (medication), diagnosis and medication intake (medical history and medication), and diagnosis and non-diagnosis with medication intake (medical history or normal with medication) alternatively defined the factor hypertension.

\section{Hypercholesterolemia}

Positive medical history and/or intake of lipid-lowering medication were considered for factor definitions.

\section{Cardiovascular disease/cardiac conditions}

Life-time diagnosis of atherosclerosis, atrial fibrillation, cardiac arrhythmia, myocardial infarction, congestive heart failure, and coronary heart disease, were each independent factor definitions.

\section{Obesity}

A body mass index (BMI; mass $\left.[\mathrm{kg}] /(\text { height }[\mathrm{m}])^{2}\right)$ above 30 (obese class I) and BMI > 35 (obese class II) was considered. BMI data of 10 participants were missing.

\section{Smoking}

Personal history of smoking behavior was indicated by "pack years" quantifying the packs (of 20 cigarettes) smoked per day multiplied by years as a smoker. Due to lack of consensus, we arbitrarily set the factor threshold to 5, 10, 15, and 20 pack years as factor definitions. For 40 former smokers, pack years data were not available.

Due to lack of vascular factor data the VBI could not be calculated for a total of 50 individuals.

\section{ULTRASONOGRAPHY}

We performed ultrasonographic measurements of the right CCA using an ACUSON Antares ${ }^{\mathrm{TM}}$ ultrasound system (Siemens, Erlangen, Germany) and a 5-10 MHz linear array transducer (VF10-5, 
Table 1 | Multiple regression analyses of intima-media thickness (IMT) and single putative vascular burden factors.

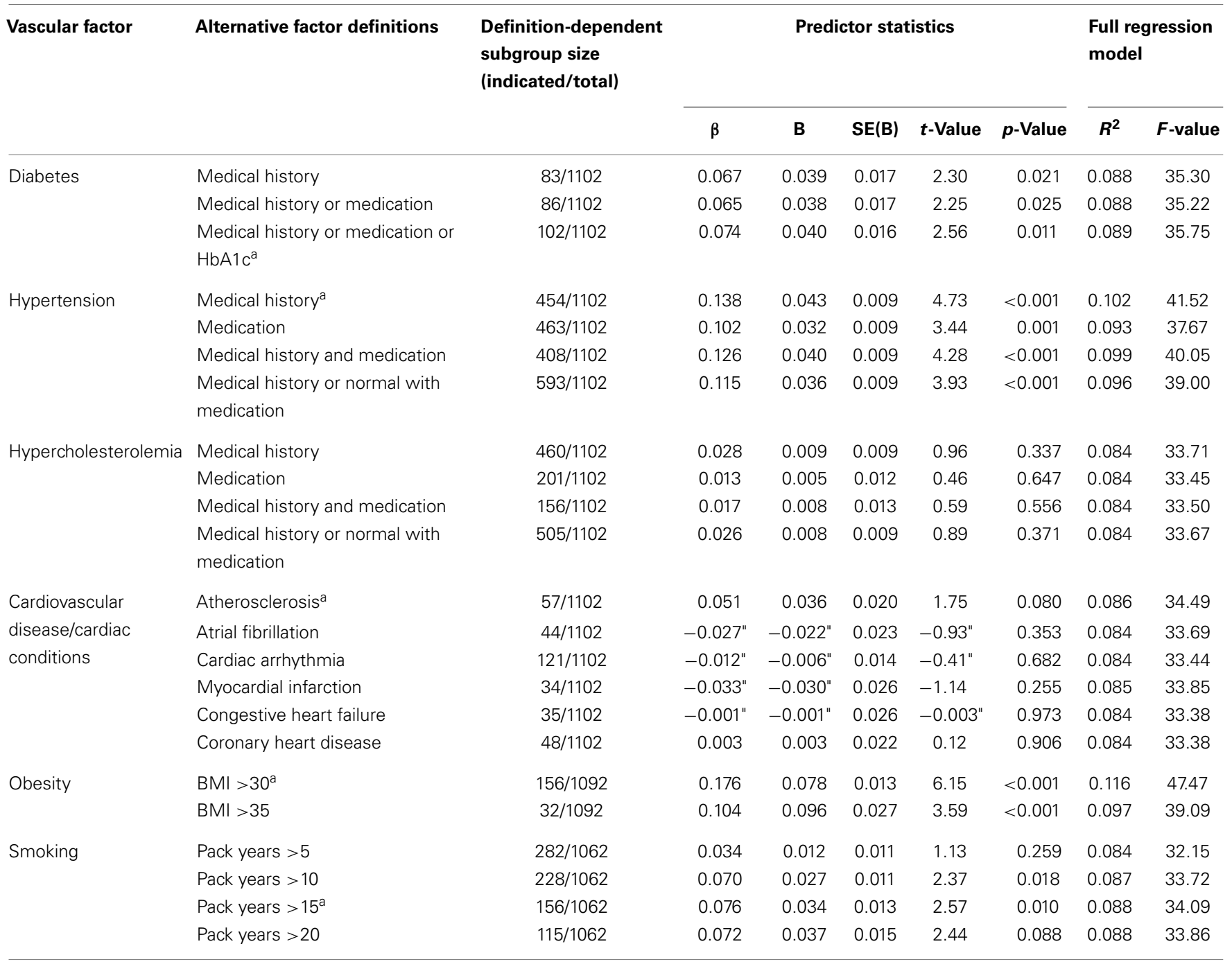

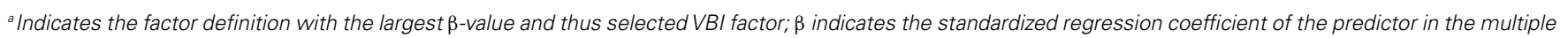
regression model; $B$ indicates the non-standardized $\beta$; and $S E(B)$ its standard error.

Siemens, Erlangen, Germany). Participants were examined in a supine position. The CCA was differentiated from the internal jugular vein in a transverse plane and then displayed in a longitudinal scan while the course of the CCA was followed to the carotid bulb. The IMT of the CCA was defined as the distance between the echogenic intima-line and the echogenic media-adventitia border at the far-wall of the CCA (Touboul et al., 2012). IMT was measured $1 \mathrm{~cm}$ proximal to the carotid bulb in a segment free of plaque using a fourfold magnification of the ultrasound image (Engelen et al., 2012). After visual inspection image quality at the site of measurement was verified by an experienced sonographer.

\section{COGNITIVE TESTING}

Participants performed established and standardized cognitive tests including the Trail-Making Test (TMT; Corrigan and Hinkeldey, 1987; Bowie and Harvey, 2006; Sanchez-Cubillo et al., 2009) and the consortium to establish a registry for Alzheimer's disease (CERAD) neuropsychological battery (Morris et al., 1989).
The administered TMT is a paper-and-pencil task and consists of two parts: for the TMT Part A participants connect randomly distributed numbers (1-25) in ascending numerical order. For the TMT Part B participants connect randomly distributed numbers (from 1 to 13 ) and letters (from $A$ to $L$ ) in alternating numeric and alphabetical order $(1-\mathrm{A}-2-\mathrm{B}-3-\mathrm{C}-\ldots-13-\mathrm{L})$. In case of errors, immediate feedback is provided by the examiner, so that the participant completes the task without errors (at the expense of additional time) (Bowie and Harvey, 2006). TMT performance was indicated by the time $[\mathrm{s}]$ for completing TMT Part B minus the time needed for TMT Part A. The difference TMT B-A represents an effective performance measure of cognitive control and executive functioning, where potential bias due to differences in upper extremity motor speed, simple sequencing, visual scanning, and psychomotor functioning are minimized (Corrigan and Hinkeldey, 1987; Sanchez-Cubillo et al., 2009).

The CERAD is composed of five subtests including animal naming, modified Boston Naming Test, Mini-Mental State 
Examination (MMSE), constructional praxis, and word list memory, which have been shown to represent valid and reliable measures of cognitive performance in the elderly and in $\mathrm{AD}$ patients (Welsh-Bohmer and Mohs, 1997). The total score of the CERAD battery has been shown to provide an effective global measure of cognitive functioning for the English as well as for the German version of the CERAD used in the present study (Chandler et al., 2005; Ehrensperger et al., 2010). The total score ranges between 0 and 100 and comprises the raw scores of the CERAD animal naming (verbal fluency; maximum of 24 points), modified Boston Naming Test (15 points), word list learning (30 points), constructional praxis (11 points), word list recall (10 points), and word list recognition discriminability (10 points) (Chandler et al., 2005; Ehrensperger et al., 2010).

\section{STATISTICS}

First, we aimed to identify vascular burden factors (with the definition yielding the largest effect size), which predict IMT independent of age and sex. Therefore, we calculated multiple regressions (inclusion algorithm) with IMT as criterion variable, and age, sex (dummy variable: female $=0$, male $=1$ ), and the vascular burden factor of interest as predictors. Vascular burden factors showing a statistical trend $(p<0.1)$ of prediction of increased IMT were included in the VBI. For these factors the definition/threshold of the highest effect size as measured by the standardized $\beta$ coefficient of the predictor was chosen.

Second, we investigated the association of the VBI with (pathological) IMT extremes. Current guidelines suggest an IMT of $0.9 \mathrm{~mm}$ as a conservative estimate of common carotid IMT abnormalities (Mancia et al., 2007). Therefore, we calculated the odds ratio (OR) and the $95 \%$ confidence interval (CI) comparing individuals without vascular burden factors with individuals possessing 1 or $2+$ factors, respectively, and their frequency of falling into the $75 \%$ (IMT $<0.9 \mathrm{~mm}$ ) and $90 \%$ percentile (IMT $<1.0 \mathrm{~mm}$ ) of the IMT distribution of the sample.

Third, we analyzed the impact of the VBI on cognitive performance. Univariate analyses of covariance using the TMT $\left(\log _{10}[\mathrm{TMT} \mathrm{B}-\mathrm{A}]\right.$; logarithmized due to non-normality) or the CERAD total score as dependent variables, the VBI (0, $1,2+$ vascular burden factors) as between-subject factor, and age, sex, and education as covariates were performed. Education level was coded according to the International Standard Classification of Education (ISCED-97) of the UNESCO (http: //www.unesco.org/) as low (level 1-2; primary education and secondary education first stage), medium (3-4; secondary and post-secondary, non-tertiary education), and high (5-6; tertiary education).

Post hoc $t$-tests were performed and the significance threshold was set to $\alpha<5 \%$, and to $\alpha<10 \%$ for statistical trends for all tests.

\section{RESULTS}

\section{SAMPLE CHARACTERISTICS}

The mean age $( \pm S D)$ of the cohort was $65.1 \pm 6.8$ years (range: $50.8-83.7$ years). Compared to males (66.0 $\pm 6.7 ; 51.6-$ $82.2)$, females $(64.4 \pm 6.8$; range: $50.8-83.7)$ were younger $\left(t_{1100}=-4.09, p<0.001\right)$. Males had higher education levels (low: $0.9 \%$, medium: $49.5 \%$, and high: $49.5 \%$ ) than females $\left(11.2 \%, 61.4 \%\right.$, and $\left.27.5 \% ; \chi^{2}=91.55, p<0.001\right)$.

\section{INTIMA-MEDIA THICKNESS}

The IMT had a mean value of $0.76 \pm 0.16 \mathrm{~mm}$. Females had lower IMT values than males $(0.74 \pm 0.15 \mathrm{~mm}$ versus $0.77 \pm 0.16 \mathrm{~mm}$, $\left.t_{1100}=-4.00, p<0.001\right)$.

\section{EFFECTS OF SINGLE VASCULAR FACTORS ON IMT}

The prevalence of the vascular burden factors is given in Table 1 . IMT was significantly predicted by age (mean $\beta$-value $( \pm S D)$ of the 23 multiple regressions: $0.262 \pm 0.011, p<0.001$ ) as well as sex $(0.087 \pm 0.006, p<0.05)$.

Independent of these age and sex effects several vascular burden factors significantly predicted IMT. Obesity $($ BMI $>30)$ was the strongest IMT predictor $(\beta=0.176)$, followed by hypertension (medical history; $\beta=0.138$ ), smoking ( pack years $>15 ; \beta=0.076$ ), and diabetes (medical history/medication $+\mathrm{HbA}_{1 c} ; \beta=0.074$ ), while atherosclerosis showed a statistical trend of IMT prediction $(\beta=0.051, p=0.080)$. These vascular burden factors were therefore selected to be included in the VBI. The full regression models [including the predictors age, sex, and single (significant) vascular burden factors] explained between 8.6 and $11.6 \%$ of the IMT variance.

No significant IMT prediction was found for other cardiovascular diseases/conditions $(p>0.3)$ or hypercholesterolemia $(p>0.3)$ in regression models including age and sex as predictors.

\section{VASCULAR BURDEN INDEX IN ASSOCIATION WITH IMT}

The number of selected vascular burden factors (VBI) showed an effect size of $\beta=0.203$ with regard to IMT prediction independent of age and sex. The full model (age, sex, VBI) explained $12.4 \%$ of IMT variance. For the VBI groups $(0,1,2+$ vascular burden factors), the mean IMT values corrected for age and sex are shown in Figure 1. The VBI was correlated with age (Spearman $\rho=0.11$, $p<0.001$ ), but did not differ between males and females (logistic regression including age; $p=0.53$ ).

After selection of the single vascular burden factors on the basis of their effects on IMT the impact of the cumulative number of these factors (VBI) on pathological IMT values was investigated.

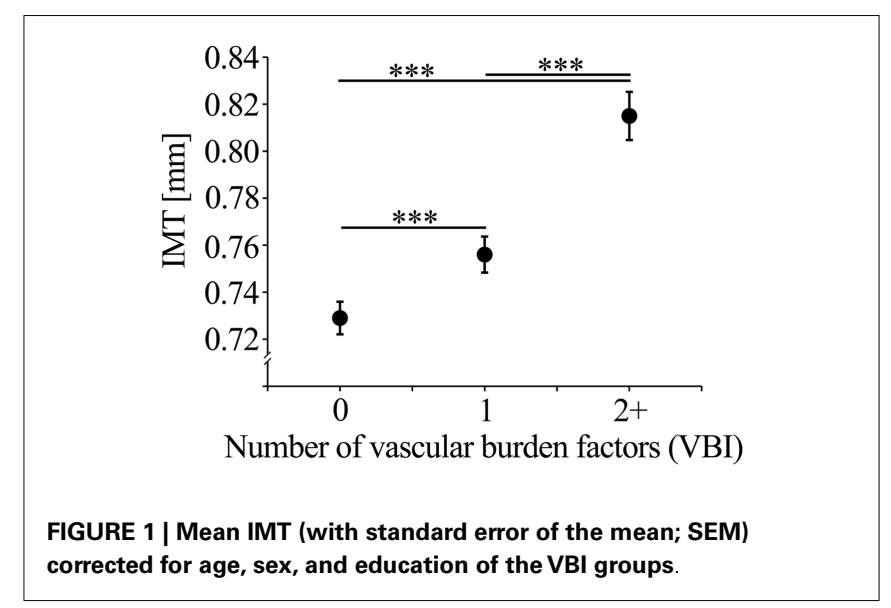


The VBI was significantly related to the frequency of individuals being in the upper $25 \%\left(\geq 0.9 \mathrm{~mm} ; \chi^{2}=35.77, p<0.001\right)$ or the upper $10 \%\left(\geq 1.0 \mathrm{~mm} ; \chi^{2}=27.62, p<0.001\right)$ of the IMT distribution. Individuals with one compared with those without vascular burden factors had an OR of 1.68 (95\% CI: 1.21-2.33) of being in the upper $25 \%$ of the IMT distribution. However, for individuals with two or more compared to those without vascular burden factors an increased OR for the upper 25\% of 2.97 (95\% CI: 2.064.28), and 3.17 (95\% CI: 1.95-5.16) for the upper $10 \%$ of the IMT distribution was observed.

\section{VASCULAR BURDEN INDEX IN ASSOCIATION WITH COGNITIVE PERFORMANCE}

The VBI significantly impacted cognitive control and executive function as indicated by TMT performance $\left(F_{2,1033}=3.08\right.$, $p=0.047)$. Post hoc tests showed that individuals with two or more vascular burden factors showed lower TMT performance (i.e., longer time for TMT B-A) compared to individuals without these factors $(p=0.015)$. Compared to those individuals with one vascular burden factor this performance difference showed a statistical trend $(p=0.051)$. Mean values of TMT performance corrected for age, sex, and education are shown in Figure 2A.

Global cognitive functioning as indicated by the CERAD total score showed a statistical trend of an impact of the VBI $\left(F_{2,1043}=2.88, p=0.057\right)$. Individuals with two or more vascular burden factors showed decreased global cognitive performance compared to individuals without $(p=0.030)$, or with one vascular burden factor $(p=0.028)$. CERAD total score mean values corrected for age, sex, and education are shown in Figure 2B.

Unlike the VBI, the presence of IMT pathology (i.e., IMT $\geq 0.9$ and IMT $\geq 1.0$, respectively) showed no significant impact on cognitive performance measures $(p>0.10)$ independent of age, sex, and education.

\section{DISCUSSION}

In the present study, a VBI based on vascular risk factors previously associated with $\mathrm{AD}$ was established for a large cohort of 1102 elderly individuals at risk for neurodegenerative diseases (TREND study). Based on the impact of single (cardio)vascular risk factors on IMT vascular burden factors were selected to be included in a cumulative VBI. Moreover, the relevance of this VBI for IMT extremes as well as for cognition in the TREND cohort was investigated.

\section{SINGLE VASCULAR BURDEN FACTORS}

The cumulative VBI established in this study comprises the factors obesity (standardized $\beta=0.176)$, hypertension $(\beta=0.138)$, diabetes $(\beta=0.074)$, smoking $(\beta=0.076)$, and atherosclerosis $(\beta=0.051)$, which predicted IMT independent of age $(\beta=0.262)$ and sex $(\beta=0.087)$. However, effect sizes were small and together with age and sex the single factors only explained between 8.6 and $11.6 \%$ of the IMT variance.

Previous studies investigating individuals in a similar age range have consistently reported an impact of these single vascular factors on IMT. For age comparable effect sizes have been shown

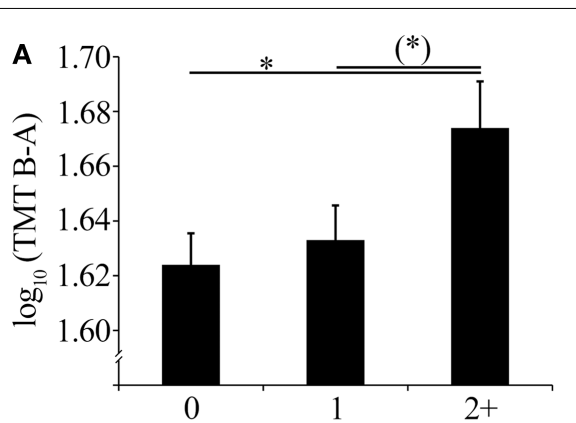

Number of vascular burden factors (VBI)

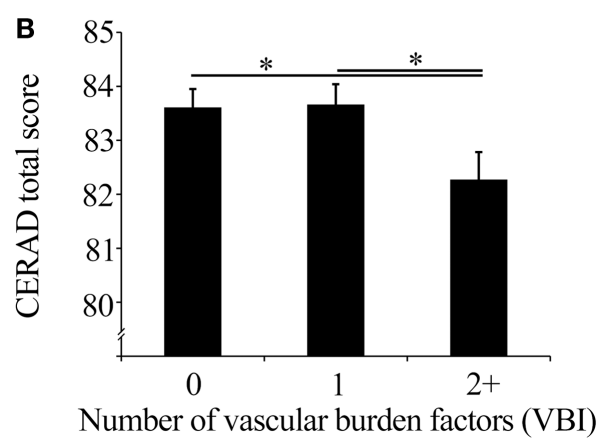

FIGURE 2 | (A) Mean TMT performance (with SEM) corrected for age, sex, and education of the VBI groups, and (B) mean CERAD total scores (with SEM) corrected for age, sex, and education of the VBI groups.

$(\beta=0.276)$ (Rundek et al., 2013). Also, males have been shown to have slightly higher IMT values than females $(\beta=0.150)$ (Rundek et al., 2013).

For the BMI, a similar effect size of IMT prediction $(\beta \sim 0.1)$ has been reported for elderly adults (Engelen et al., 2012). An IMT difference of about $0.1 \mathrm{~mm}$ between adult normal-weighted and obese individuals has been previously reported (Kotsis et al., 2006), which corresponds to the effects of class I obesity $(\mathrm{BMI}>30)$ in the present sample.

The small effect size of life-time diagnosis of hypertension in the present sample is comparable to the previously reported small effect size of IMT prediction by systolic blood pressure $(\beta \sim 0.1)$ (Engelen et al., 2012), or IMT differences between elderly normotensive and hypertensive individuals (Bots et al., 1993).

For diabetes predicting increased IMT similar effect sizes in elderly individuals with cardiovascular diseases $(\beta \sim 0.10)$ and a slightly higher effect size $(\beta \sim 0.19)$ in a healthy elderly population has been reported (Engelen et al., 2012).

Smoking as defined by more than 15 pack years showed a slightly smaller effect size as previously reported for pack years smoking $(\beta=0.125)$ (Salonen and Salonen, 1991). However, for current smoking effect sizes were shown to differ between healthy, elderly individuals and sexes (men: $\beta=0.25$, women: $\beta=0.11$ ) and elderly individuals with cardiovascular diseases (men: $\beta=0.10$, women: $\beta=-0.10$ ) (Engelen et al., 2012). Thus, the impact of the factor smoking on IMT might be complex and sex-dependent. 
Contrary to numerous previous studies associating atherosclerosis with increased IMT (Bots et al., 1996, 1997; van Popele et al., 2001), we only found a small effect of the factor atherosclerosis $(\beta=0.051)$ on IMT. However, effect sizes of reported life-time atherosclerosis $(n=57)$ may be small as effects on IMT prediction were calculated to be independent of age and sex.

Other cardiovascular diseases and conditions were no significant IMT predictors in the present study, which is in contrast to several previous findings showing increased IMT for e.g., myocardial infarction, congestive heart failure, coronary heart disease (Bots et al., 1997; Den Ruijter et al., 2012; van den Oord et al., 2013). The missing IMT effects in the present study might be due to the younger age and rather small number of individuals with these cardiovascular pathologies, and age and sex being confounding factors in multiple regression analyses.

Life-time diagnosis of hypercholesterolemia was no significant IMT predictor independent of age and sex in the present study. Recently, very small effects $(\beta<0.05)$ of IMT prediction have been reported for the Total-to-HDL cholesterol ratio in elderly individuals (Engelen et al., 2012). Also, life-time hypercholesterolemia effects on IMT might have been ameliorated due to lipid-lowering treatment or nutritional changes in the past.

\section{VASCULAR BURDEN INDEX}

The number of vascular burden factors (VBI) together with age and sex explained $12.4 \%$ of the IMT variance. This percentage corresponds to previous large studies in elderly individuals, which showed 11\% (Rundek et al., 2013), 17\% (O'Leary et al., 1996), or $27 \%$ (Baldassarre et al., 2010) of IMT variance to be explained by age, sex, and vascular burden factors (e.g., blood pressure, pack years or current smoking, serum cholesterol or glucose levels, and others) and factors, such as education or geographical data. However, composition of these factors included in these studies did widely differ, and in the study by Baldassarre et al. (2010) the IMT predictor with the largest effect size was geographical latitude within Europe (partial $r=0.078$ ), which partly explains the large percentage $(27 \%)$ of explained IMT variance.

Thus, variance of IMT as a continuous variable including non-pathological IMT values $(<0.9 \mathrm{~mm})$ and (heritable) natural differences between individuals might only be modestly explained by vascular burden factors. This might also underlie the modestly increased IMT prediction comparing the VBI (12.4\%) and the single vascular factor of largest effect size (obesity: 11.6\%). However, the impact of vascular burden factors might be better observed for pathological IMT extremes. Here, we found a pronounced impact of the number of vascular burden factors with respect to the extreme subgroups. For instance, individuals with two or more compared to those without vascular burden factors showed an increased OR of 2.97 for IMT $\geq 0.9 \mathrm{~mm}$, and an OR of 3.17 for IMT $\geq 1.0 \mathrm{~mm}$. These findings suggest that within normal, non-pathological IMT ranges (i.e., IMT $<0.9 \mathrm{~mm}$ ) natural and largely age- and sex-dependent interindividual IMT variability might prevail, whereas the impact of the VBI is best observed in subgroups of increased IMT.

The VBI was established for the TREND cohort to investigate its predictive value for MCI and AD in future longitudinal investigations of the TREND study. Importantly, in elderly individuals, in whom cerebral perfusion is already diminished due to aging a high vascular load, e.g., mediated by two, three, or more vascular burden factors, might causally underlie chronic brain hypoperfusion, hypoxia, and a neuronal energy crisis critical with respect to cellular functioning and may, hence, foster neurodegeneration increasing the risk of AD (Li et al., 2011; de la Torre, 2012a). Because chronic brain hypoperfusion often precedes MCI and AD by many years, the present study identified individuals hypothesized to show cognitive decline in the future due to vascular burden factors. A recent study even showed a high association of increased pulse wave velocity with beta-amyloid burden in elderlies (Hughes et al., 2013) suggesting an important link between vascular and beta-amyloid mediated pathological processes relevant to AD.

\section{VASCULAR BURDEN INDEX AND COGNITION}

The present study showed an impact of the VBI on cognition. Individuals with two or more vascular burden factors exhibited poorer executive function/cognitive control in the TMT and lower global cognitive function in the CERAD neuropsychological battery compared to individuals without these factors, which was independent of age, sex, and education. This finding indicates that the VBI is indeed associated with cognitive performance in this study cohort. As associations between cognitive performance and IMT were not significant in the present study, the VBI may represent a more reliable and holistic indicator of vascular impact on cognition, compared to (pathological) IMT alone.

Importantly, the VBI comprises vascular factors which have previously been associated with $\mathrm{AD}$. Also, by considering objective and quantitative IMT measurements for the selection of these factors and factor definitions, the validity of the VBI indicating vascular burden as relevant for AD in individuals of the TREND cohort might be increased. Thus, the VBI may hold important predictive value for cognitive decline and the development of MCI and $\mathrm{AD}$, which will be further investigated in the longitudinal TREND study.

\section{LIMITATIONS}

Several limitations have to be considered for the present study: (1) by selecting vascular factors for the VBI based on IMT some putative factors/definitions were not included in the VBI. This procedure may increase sensitivity of the VBI as selected factors actually predict increased IMT in the TREND cohort but may also reduce its specificity by excluding factors (e.g., cardiovascular pathologies, hypercholesterolemia). (2) We only used questionnaires and personal medical history interviews for the assessment of vascular risk factors, diseases, and medication data. However, it was emphasized that all indications, diagnoses, and medications must have been confirmed by a medical doctor. Moreover, experimental blood pressure measurements were not included in the establishment of the VBI and the identification of individuals with increased vascular burden. Reliable (multiple) measurements of blood pressure or other experimental vascular and laboratory measures should be investigated in future studies for their potential to further validate or refine vascular burden estimates, such as the VBI. (3) While total or HDL cholesterol, or fasting glucose levels or other blood-derived quantitative measures were not assessed due to assessments at different day times, we aimed to 
complement the data by also considering current medication and, for diabetes, blood $\mathrm{HbA}_{1 \mathrm{c}}$ concentrations. (4) We only assessed one frequently investigated segment of the IMT of the right CCA as a reference measure of vascular burden, as the design of the TREND study favors easy to apply and quick to perform techniques, which may well be used in other large studies or populations screenings. However, other indicators including internal carotid artery measures (Mackinnon et al., 2004), regional plaques (Zureik et al., 2002), and arterial stiffness (van Popele et al., 2001; Hughes et al., 2013) were not investigated, but might also be important for the relationship between vascular burden factors and vascular functionality. (5) Interactions between vascular burden factors were not in the scope of the present study. However, their interactions have been shown to only modestly improve explained IMT variance (Niu et al., 2013). (6) The present results might not be fully generalizable, as TREND study participants had above average education levels (39\% academics) and were partly selectively included due to the presence of prodromal markers for neurodegeneration (REM-sleep disorder, hyposmia, and/or depression) (Berg, 2012).

\section{CONCLUSION}

Vascular function and structure as well as the risk of AD have been shown to be influenced by a multitude of vascular factors. Based on their impact on carotid IMT vascular factors associated with $\mathrm{AD}$ can be selected to establish a neurodegenerative cumulative VBI. The relevance of this VBI for cognition was validated by its impact on cognitive performance in healthy, non-demented individuals at risk for neurodegeneration. The VBI may represent an important predictor of $\mathrm{AD}$ and its prodromal stages, which will be further investigated in the longitudinal TREND study.

\section{AUTHOR CONTRIBUTIONS}

Conceived and designed the experiments and edited the manuscript: Gerhard W. Eschweiler, Andreas J. Fallgatter, Walter Maetzler, Daniela Berg. Performed the experiments: Benjamin Roeben, Isabel Wurster, Kathrin Brockmann, Raphael Niebler, Florian G. Metzger, Andreas Fritsche. Analyzed the data: Sebastian Heinzel, Inga Liepelt-Scarfone, Benjamin Roeben, Ulrike Suenkel, Isabella Nasi-Kordhishti. Wrote the manuscript: Sebastian Heinzel, Inga Liepelt-Scarfone, Walter Maetzler, Daniela Berg. All authors were involved in interpretation of the data and critical revision of the manuscript, all authors gave their final approval.

\section{ACKNOWLEDGMENTS}

We thank the participants and their families for their help and participation. Moreover, we especially thank Dr. Jana Godau and Dr. Heiko Huber for their support of the ultrasound measurements, Heide Schmidt for organizing the participant recruitment of the TREND study (first follow-up), and Alexandra Taylor for proofreading the manuscript for language issues. Moreover, we acknowledge the contributions of all members of the TREND study consortium (March 2011 to April 2012). This study was supported by intramural funding as well as by the German Center for Neurodegenerative Diseases (DZNE). Kathrin Brockmann has received honoraria for lectures from the Deutsche Gesellschaft für klinische Neurophysiologie (DGKN) as well as travel grants from GlaxoSmithKline, UCB, and the Movement Disorders Society. Gerhard W. Eschweiler has received grants for phase II and phase III studies in Alzheimer disorders from AC Immune and Janssen Alzheimer Immunotherapy Research. Andreas J. Fallgatter has received research support from the German Research Foundation, German Ministry for Education and Research, Center of Integrative Neurosciences, from AstraZeneca for an investigator-initiated trial. Walter Maetzler has received research support from the Robert Bosch Foundation and speaker honoraria from GlaxoSmithKline, and research support from the European Union, the Michael Fox Foundation, and from Janssen Pharmaceutica. Daniela Berg has received Advisory Boards from UCB Schwarz Pharma, Novartis, Merz; honoraria from UCB Schwarz Pharma, GSK, TEVA, Lundbeck; grants from Michael J. Fox Foundation, BmBF, Janssen Pharmaceutica, TEVA Pharma GmbH, Böhringer, dPV (German Parkinson's disease association), Abbott, and Center of Integrative Neurosciences.

\section{REFERENCES}

Baldassarre, D., Nyyssonen, K., Rauramaa, R., De Faire, U., Hamsten, A., Smit, A. J., et al. (2010). Cross-sectional analysis of baseline data to identify the major determinants of carotid intima-media thickness in a European population: the IMPROVE study. Eur. Heart J. 31, 614-622. doi:10.1093/eurheartj/ehp496

Berg, D. (2012). Is pre-motor diagnosis possible? The European experience. Parkinsonism Relat. Disord. 18(Suppl. 1), S195-S198. doi:10.1016/S1353-8020(11) 70061-X

Bots, M. L., Hoes, A. W., Koudstaal, P. J., Hofman, A., and Grobbee, D. E. (1997). Common carotid intima-media thickness and risk of stroke and myocardial infarction - the Rotterdam study. Circulation 96, 1432-1437. doi:10.1161/01. CIR.96.5.1432

Bots, M. L., Hofman, A., De Bruyn, A. M., De Jong, P. T., and Grobbee, D. E. (1993). Isolated systolic hypertension and vessel wall thickness of the carotid artery. The Rotterdam elderly study. Arterioscler. Thromb. 13, 64-69. doi:10.1161/01.ATV.13.1.64

Bots, M. L., Hofman, A., Dejong, P. T. V. M., and Grobbee, D. E. (1996). Common carotid intima media thickness as an indicator of atherosclerosis at other sites of the carotid artery - the Rotterdam study. Ann. Epidemiol. 6, 147-153. doi:10.1016/1047-2797(96)00001-4

Bowie, C. R., and Harvey, P. D. (2006). Administration and interpretation of the trail making test. Nat. Protoc. 1, 2277-2281. doi:10.1038/nprot.2006.390

Breteler, M. M. B. (2000). Vascular risk factors for Alzheimer's disease: an epidemiologic perspective. Neurobiol. Aging 21, 153-160. doi:10.1016/S0197-4580(99) 00110-4

Cataldo, J. K., Prochaska, J. J., and Glantz, S. A. (2010). Cigarette smoking is a risk factor for Alzheimer's disease: an analysis controlling for tobacco industry affiliation. J. Alzheimers Dis. 19, 465-480. doi:10.3233/JAD-2010-1240

Chandler, M. J., Lacritz, L. H., Hynan, L. S., Barnard, H. D., Allen, G., Deschner, M., et al. (2005). A total score for the CERAD neuropsychological battery. Neurology 65, 102-106. doi:10.1212/01.wnl.0000167607.63000.38

Chao, L. L., Buckley, S. T., Kornak, J., Schuff, N., Madison, C., Yaffe, K., et al. (2010). ASL perfusion MRI predicts cognitive decline and conversion from MCI to dementia. Alzheimer Dis. Assoc. Disord. 24, 19-27. doi:10.1097/WAD. 0b013e3181b4f736

Corrigan, J. D., and Hinkeldey, N. S. (1987). Relationships between parts A and B of the trail making test. J. Clin. Psychol. 43, 402-409. doi:10.1002/10974679(198707)43:4<402::AID-JCLP2270430411>3.0.CO;2-E

D’Agostino, R. B. Sr., Vasan, R. S., Pencina, M. J., Wolf, P. A., Cobain, M., Massaro, J. M., et al. (2008). General cardiovascular risk profile for use in primary care: the Framingham heart study. Circulation 117, 743-753. doi:10.1161/ CIRCULATIONAHA.107.699579

de la Torre, J. C. (2012a). Cardiovascular risk factors promote brain hypoperfusion leading to cognitive decline and dementia. Cardiovasc. Psychiatry. Neurol. 2012, 367516. doi:10.1155/2012/367516 
de la Torre, J. C. (2012b). Cerebral hemodynamics and vascular risk factors: setting the stage for Alzheimer's disease. J. Alzheimers Dis. 32, 553-567. doi:10.3233/ JAD-2012-120793

Den Ruijter, H. M., Peters, S. A. E., Anderson, T. J., Britton, A. R., Dekker, J. M., Eijkemans, M. J., et al. (2012). Common carotid intima-media thickness measurements in cardiovascular risk prediction: a meta-analysis. JAMA 308, 796-803. doi:10.1001/jama.2012.9630

Ehrensperger, M. M., Berres, M., Taylor, K. I., and Monsch, A. U. (2010). Early detection of Alzheimer's disease with a total score of the German CERAD. J. Int Neuropsychol. Soc. 16, 910-920. doi:10.1017/S1355617710000822

Engelen, L., Ferreira, I., Stehouwer, C. D., Boutouyrie, P., and Laurent, S. (2012). Reference intervals for common carotid intima-media thickness measured with echotracking: relation with risk factors. Eur. Heart J. 34, 2368-2380. doi:10.1093/eurheartj/ehs380

Folsom, A. R., Eckfeldt, J. H., Weitzman, S., Ma, J., Chambless, L. E., Barnes, R. W., et al. (1994). Relation of carotid-artery wall thickness to diabetes-mellitus, fasting glucose and insulin, body-size, and physical-activity. Stroke 25, 66-73. doi:10.1161/01.STR.25.1.66

Gaenslen, A., Wurster, I., Brockmann, K., Huber, H., Godau, J., Faust, B., et al. (2014). Prodromal features for Parkinson's disease - baseline data from the TREND study. Eur. J. Neurol. 21, 766-772. doi:10.1111/ene.12382

Heinzel, S., Metzger, F. G., Ehlis, A. C., Korell, R., Alboji, A., Haeussinger, F. B., et al. (2013). Aging-related cortical reorganization of verbal fluency processing: a functional near-infrared spectroscopy study. Neurobiol. Aging 34, 439-450. doi:10.1016/j.neurobiolaging.2012.05.021

Hobert, M. A., Niebler, R., Meyer, S. I., Brockmann, K., Becker, C., Huber, H., et al. (2011). Poor trail making test performance is directly associated with altered dual task prioritization in the elderly - baseline results from the TREND study. PLoS ONE 6:e27831. doi:10.1371/journal.pone.0027831

Honig, L. S., Tang, M. X., Albert, S., Costa, R., Luchsinger, J., Manly, J., et al. (2003). Stroke and the risk of Alzheimer disease. Arch. Neurol. 60, 1707-1712. doi:10.1001/archneur.60.12.1707

Hort, J., O’brien, J. T., Gainotti, G., Pirttila, T., Popescu, B. O., Rektorova, I., et al. (2010). EFNS guidelines for the diagnosis and management of Alzheimer's disease. Eur. J. Neurol. 17, 1236-1248. doi:10.1111/j.1468-1331.2010.03040.x

Hughes, T. M., Kuller, L. H., Barinas-Mitchell, E. J., Mackey, R. H., Mcdade, E. M., Klunk, W. E., et al. (2013). Pulse wave velocity is associated with betaamyloid deposition in the brains of very elderly adults. Neurology 81, 1711-1718. doi:10.1212/01.wnl.0000435301.64776.37

Kilpatrick, E. S., Bloomgarden, Z. T., and Zimmet, P. Z. (2009). International expert committee report on the role of the AlC assay in the diagnosis of diabetes: response to the international expert committee. Diabetes Care 32, e159. doi:10.2337/dc09-1231

Kivipelto, M., Ngandu, T., Fratiglioni, L., Viitanen, M., Kareholt, I., Winblad, B., et al. (2005). Obesity and vascular risk factors at midlife and the risk of dementia and Alzheimer disease. Arch. Neurol. 62, 1556-1560. doi:10.1001/archneur. 62.10 .1556

Kivipelto, M., and Solomon, A. (2006). Cholesterol as a risk factor for Alzheimer's disease - epidemiological evidence. Acta Neurol. Scand. Suppl. 185, 50-57. doi:10.1111/j.1600-0404.2006.00685.x

Kotsis, V. T., Stabouli, S. V., Papamichael, C. M., and Zakopoulos, N. A. (2006). Impact of obesity in intima media thickness of carotid arteries. Obesity (Silver Spring) 14, 1708-1715. doi:10.1038/oby.2006.196

Langbaum, J. B., Fleisher, A. S., Chen, K., Ayutyanont, N., Lopera, F., Quiroz, Y. T., et al. (2013). Ushering in the study and treatment of preclinical Alzheimer disease. Nat. Rev. Neurol. 9, 371-381. doi:10.1038/nrneurol.2013.107

Li, J., Wang, Y. J., Zhang, M., Xu, Z. Q., Gao, C. Y., Fang, C. Q., et al. (2011). Vascular risk factors promote conversion from mild cognitive impairment to Alzheimer disease. Neurology 76, 1485-1491. doi:10.1212/WNL.0b013e318217e7a4

Lim, Y. Y., Maruff, P., Pietrzak, R. H., Ellis, K. A., Darby, D., Ames, D., et al. (2014). Abeta and cognitive change: examining the preclinical and prodromal stages of Alzheimer's disease. Alzheimers Dement. doi:10.1016/j.jalz.2013.11.005

Lorenz, M. W., Markus, H. S., Bots, M. L., Rosvall, M., and Sitzer, M. (2007). Prediction of clinical cardiovascular events with carotid intima-media thickness: a systematic review and meta-analysis. Circulation 115, 459-467. doi:10.1161/ CIRCULATIONAHA.106.628875

Mackinnon, A. D., Jerrard-Dunne, P., Sitzer, M., Buehler, A., Von Kegler, S., and Markus, H. S. (2004). Rates and determinants of site-specific progression of carotid artery intima-media thickness: the carotid atherosclerosis progression study. Stroke 35, 2150-2154. doi:10.1161/01.STR.0000136720.21095.f3

Maetzler, W., Langkamp, M., Lerche, S., Godau, J., Brockmann, K., Gaenslen, A., et al (2012). Lowered serum amyloid-beta1-42 autoantibodies in individuals with lifetime depression. J. Alzheimers Dis. 32, 95-100. doi:10.3233/JAD-2012-120625

Mancia, G., De Backer, G., Dominiczak, A., Cifkova, R., Fagard, R., Germano, G., et al. (2007). 2007 Guidelines for the management of arterial hypertension: the task force for the management of arterial hypertension of the European society of hypertension (ESH) and of the European society of cardiology (ESC). Eur. Heart J. 28, 1462-1536. doi:10.1093/eurheartj/ehm236

Moghekar, A., Li, S., Lu, Y., Li, M., Wang, M. C., Albert, M., et al. (2013). CSF biomarker changes precede symptom onset of mild cognitive impairment. Neurology 81, 1753-1758. doi:10.1212/01.wnl.0000435558.98447.17

Morris, J. C., Heyman, A., Mohs, R. C., Hughes, J. P., Van Belle, G., Fillenbaum, G., et al. (1989). The consortium to establish a registry for Alzheimer's disease (CERAD). Part I. Clinical and neuropsychological assessment of Alzheimer's disease. Neurology 39, 1159-1165. doi:10.1212/WNL.39.9.1159

Niu, L. L., Zhang, Y. L., Qian, M., Meng, L., Xiao, Y., Wang, Y. Y., et al. (2013). Impact of multiple cardiovascular risk factors on carotid intima-media thickness and elasticity. PLoS ONE 8:e67809. doi:10.1371/journal.pone.0067809

O’Leary, D. H., Polak, J. F., Kronmal, R. A., Savage, P. J., Borhani, N. O., Kittner, S. J., et al. (1996). Thickening of the carotid wall. A marker for atherosclerosis in the elderly? Cardiovascular Health Study Collaborative Research Group. Stroke 27, 224-231. doi:10.1161/01.STR.27.2.224

Olesen, J. B., Lip, G. Y., Hansen, M. L., Hansen, P. R., Tolstrup, J. S., Lindhardsen, J., et al. (2011). Validation of risk stratification schemes for predicting stroke and thromboembolism in patients with atrial fibrillation: nationwide cohort study. BMJ 342, d124. doi:10.1136/bmj.d124

Purnell, C., Gao, S., Callahan, C. M., and Hendrie, H. C. (2009). Cardiovascular risk factors and incident Alzheimer disease: a systematic review of the literature. Alzheimer Dis. Assoc. Disord. 23, 1-10. doi:10.1097/WAD.0b013e318187541c

Rundek, T., Blanton, S. H., Bartels, S., Dong, C., Raval, A., Demmer, R. T., et al. (2013). Traditional risk factors are not major contributors to the variance in carotid intima-media thickness. Stroke 44, 2101-2108. doi:10.1161/STROKEAHA.111. 000745

Salonen, R., and Salonen, J. T. (1991). Determinants of carotid intima-media thickness: a population-based ultrasonography study in eastern Finnish men. J. Intern. Med. 229, 225-231. doi:10.1111/j.1365-2796.1991.tb00336.x

Sanchez-Cubillo, I., Perianez, J. A., Adrover-Roig, D., Rodriguez-Sanchez, J. M., Rios-Lago, M., Tirapu, J., et al. (2009). Construct validity of the trail making test: role of task-switching, working memory, inhibition/interference control, and visuomotor abilities. J. Int. Neuropsychol. Soc. 15, 438-450. doi:10.1017/ S1355617709090626

Smith, C. D., Andersen, A. H., Gold, B. T., and Neuroimaging, A. D. (2012). Structural brain alterations before mild cognitive impairment in ADNI: validation of volume loss in a predefined antero-temporal region. J. Alzheimers Dis. 31, S49-S58. doi:10.3233/Jad-2012-120157

Stein, J. H., Fraizer, M. C., Aeschlimann, S. E., Nelson-Worel, J., Mcbride, P. E., and Douglas, P. S. (2004). Vascular age: integrating carotid intima-media thickness measurements with global coronary risk assessment. Clin. Cardiol. 27, 388-392. doi:10.1002/clc.4960270704

Touboul, P. J., Hennerici, M. G., Meairs, S., Adams, H., Amarenco, P., Bornstein, N., et al. (2012). Mannheim carotid intima-media thickness and plaque consensus (2004-2006-2011). An update on behalf of the advisory board of the 3rd, 4th and 5 th watching the risk symposia, at the 13th, 15th and 20th European stroke conferences, Mannheim, Germany, 2004, Brussels, Belgium, 2006, and Hamburg, Germany, 2011. Cerebrovasc. Dis. 34, 290-296. doi:10.1159/000343145

Urbina, E. M., Srinivasan, S. R., Tang, R., Bond, M. G., Kieltyka, L., and Berenson, G. S. (2002). Impact of multiple coronary risk factors on the intima-media thickness of different segments of carotid artery in healthy young adults (the Bogalusa heart study). Am. J. Cardiol. 90, 953-958. doi:10.1016/S0002-9149(02)02660-7 van den Oord, S. C. H., Sijbrands, E. J. G., Ten Kate, G. L., Van Klaveren, D., Van Domburg, R. T., Van Der Steen, A. F. W., et al. (2013). Carotid intima-media thickness for cardiovascular risk assessment: systematic review and meta-analysis. Atherosclerosis 228, 1-11. doi:10.1016/j.atherosclerosis.2013.01.025

van Popele, N. M., Grobbee, D. E., Bots, M. L., Asman, R., Topouchian, J., Reneman, R. S., et al. (2001). Association between arterial stiffness and atherosclerosis - the Rotterdam study. Stroke 32, 454-460. doi:10.1161/01.STR.32.2.454 
Welsh-Bohmer, K. A., and Mohs, R. C. (1997). Neuropsychological assessment of Alzheimer's disease. Neurology 49, S11-S13. doi:10.1212/WNL.49.3_ Suppl_3.S11

Zlokovic, B. V. (2005). Neurovascular mechanisms of Alzheimer's neurodegeneration. Trends Neurosci. 28, 202-208. doi:10.1016/j.tins.2005.02.001

Zureik, M., Temmar, M., Adamopoulos, C., Bureau, J. M., Courbon, D., Thomas, F, et al. (2002). Carotid plaques, but not common carotid intima-media thickness, are independently associated with aortic stiffness. J. Hypertens. 20, 85-93. doi:10.1097/00004872-200201000-00013

Conflict of Interest Statement: The authors declare that the research was conducted in the absence of any commercial or financial relationships that could be construed as a potential conflict of interest.
Received: 14 March 2014; accepted: 20 June 2014; published online: 09 July 2014. Citation: Heinzel S, Liepelt-Scarfone I, Roeben B, Nasi-Kordhishti I, Suenkel U, Wurster I, Brockmann K, Fritsche A, Niebler R, Metzger FG, Eschweiler GW, Fallgatter AJ, Maetzler W and Berg D (2014) A neurodegenerative vascular burden index and the impact on cognition. Front. Aging Neurosci. 6:161. doi: 10.3389/fnagi.2014.00161 This article was submitted to the journal Frontiers in Aging Neuroscience.

Copyright (c) 2014 Heinzel, Liepelt-Scarfone, Roeben, Nasi-Kordhishti, Suenkel, Wurster, Brockmann, Fritsche, Niebler, Metzger, Eschweiler, Fallgatter, Maetzler and Berg. This is an open-access article distributed under the terms of the Creative Commons Attribution License (CC BY). The use, distribution or reproduction in other forums is permitted, provided the original author(s) or licensor are credited and that the original publication in this journal is cited, in accordance with accepted academic practice. No use, distribution or reproduction is permitted which does not comply with these terms. 